side effects, and also places avoidable financial strain on the health service. An Australian group published an 'OncPal deprescribing guideline' to assist clinicians in identifying potentially inappropriate medications (PIMs), which was used to help reduce polypharmacy in patients discharged from our hospice ${ }^{[2]}$.

Methods Retrospective case note review of patients with a prognosis of six months or less discharged from our inpatient unit during two 2 month periods, was performed. PIMs were identified using the OncPal guideline and then assessed for appropriateness independently by two hospice doctors. Following baseline data collection, the hospice used posters and multidisciplinary team meetings to encourage use of the OncPal guideline in an effort to raise awareness and eradicate futile medicines.

Results Baseline data $(n=19)$ revealed that there were 61 PIMs on admission, of which $19.7 \%$ were deemed truly inappropriate by both doctors. Of these, $75 \%$ were discontinued, resulting in 0.16 truly inappropriate medications per patient on discharge. Between the 2 reviewing doctors, there was discordance over the perceived appropriateness of $19.7 \%$ of admission medications. Post-intervention data $(n=9)$ showed $54.8 \%$ of admission PIMs were considered truly inappropriate by both doctors, of which $35.3 \%$ were ceased, resulting in 1.22 truly inappropriate PIMs on discharge. However, discordance had dropped to $6.5 \%$.

Conclusions These results highlight the difficulties in managing medications in palliative patients. Whilst the OncPal deprescribing guideline may help healthcare professionals to identify PIMs, more interventions are needed to empower doctors to appropriately stop these medications, to the benefit of patients and the healthcare sector as a whole.

\section{P-100 NEEDS IDENTIFICATION AND PLANNING FOR PALLIATIVE CARE IN CHILDREN WITH LIFE-LIMITING CONDITIONS}

Archana Soman. Norfolk and Norwich University Hospital NHSFT, Norwich, UK

\subsection{6/bmjspcare-2017-00133.99}

Introduction The need for pro-active planning and delivery of multidisciplinary (MDT) care to children with life-limiting conditions (LLC) is widely acknowledged, and 'standards frameworks' have been recommended by Together for Short Lives and others. Palliative and end-of-life care are important priorities of the United Kingdom government.

Methods We conducted a retrospective case-notes audit of 20 children (8 cancer and 12 non-cancer), aged 28 days to 16 years, who had died as a direct result of a LLC, against seven pre-agreed standards. Sudden deaths, neonatal deaths and deaths within a month of diagnosis were excluded.

Results

- A 'breaking-bad-news' meeting was documented in $95 \%$ (Oncology 100\%, non-oncology 91.6\%), but this focussed on the diagnosis, and discussion of prognoses was lacking in most.

- A MDT meeting was documented in 85\% (Oncology: 100\%, non-oncology: 75\%).

- A key worker and a lead professional were both identified in 45\% (Oncology: 87.5\%, non-oncology: 16.7\%; $\mathrm{p}=0.01$ ).

- MDT assessment of palliative care needs was documented in 60\% (Oncology: 100\%, non-oncology: 33.3\%; $p=0.015$ ).
- Symptom management plans were found in 65\% (Oncology: 100\%, non-oncology: 41.7\%; $\mathrm{p}=0.0225$ ).

- Family and psycho-social needs were documented in $75 \%$ (Oncology: 100\%, non-oncology: 58.3\%).

- End-of-life care plans were documented in full in $40 \%$ (Oncology: 75\%, non-oncology: 16.7\%; $\mathrm{p}=0.0325$ ).

Recommendations and conclusion Recognition of needs and planning for palliative care in children remains sub-optimal overall, especially so in children with non-cancer LLC. This audit has led to much introspection and an acknowledgement of the need for hearts-and-minds change in clinicians' approaches. We hope to influence commissioners to develop a robust children's palliative care service with a complex-care co-ordinator, rolling MDT meetings and dedicated paediatrician time. A sub-regional working group is mapping services to needs, in order to identify further gaps. A comprehensive care pathway that will incorporate palliative and end-of-life care plans has been written, with input from parent groups.

\section{P-101 SOLSTICE: SANCUSO ${ }^{\circledR}$ IN SUPPORTIVE AND PALLIATIVE CARE; A FEASIBILITY STUDY IN PATIENTS WITH CANCER AND REFRACTORY NAUSEA AND VOMITING}

${ }^{1}$ Emma Dean, ${ }^{2}$ Richard Berman, ${ }^{1}$ Shaun Villa. ${ }^{1}$ Experimental Cancer Medicine Team, The Christie NHS Foundation Trust, Manchester, UK; ${ }^{2}$ Palliative Care Support TeamThe Christie NHS Foundation Trust, Manchester, UK

\subsection{6/bmjspcare-2017-00133.100}

Background Nausea and vomiting ( $\mathrm{N}$ and $\mathrm{V}$ ) are common, debilitating symptoms in patients with cancer, often precipitating inpatient admission for subcutaneous/intravenous antiemetics and re-hydration. Currently, there are no evidence-based solutions and treatment algorithms differ across clinical practice. Some of these patients will experience difficulty swallowing tablets and/or are unable to keep oral medications down. Treatments for patients with cancer may also reduce the ability of the intestines to absorb medicines within a tablet.

SANCUSO $^{\circledast}$ (Granisetron Transdermal System [transdermal skin patch]) is indicated for the prevention of ( $\mathrm{N}$ and $\mathrm{V})$ in patients receiving moderately and/or highly emetogenic chemotherapy regimens. The SANCUSO ${ }^{\circledast}$ patch delivers consistent, predictable levels of granisetron throughout five days with smoother daily pharmacokinetics compared to daily dosing. The role of Sancuso in patients with cancer and refractory $\mathrm{N}$ and $\mathrm{V}$ which is unrelated to chemotherapy has not been explored.

Methods An open-label, randomised feasibility study comparing Sancuso with 'physician's choice' of antiemetic in patients with cancer and refractory $\mathrm{N}$ and $\mathrm{V}$. A feasibility study is required at this juncture as standard antiemetic treatment in this patient population is undefined, and the therapeutic efficacy of Sancuso requires appraisal before embarking on a larger randomised trial. To assess feasibility, objectives have been categorised into the four domains; (i) Recruitment assess the number of patients approached, consent rate, number of eligible patients and explore the methods used to identify potential patients (ii) Patients - willingness to participate and acceptability of the intervention (iii) Clinicians' - ability to recruit, which physician's choice is selected, experience including monitoring of prescribing practice in the control 